\title{
Evolving CSP Algorithm in Predicting the Path Loss of Indoor Propagation Models
}

\author{
Anuj Agrawal \\ PEC University of Technology, \\ Chandigarh, India
}

\begin{abstract}
Constraint programming is the study of system which is based on constraints. The solution of a constraint satisfaction problem is a set of variable value assignments, which satisfies all members of the set of constraints in the CSP. In this paper the application of constraint satisfaction programming is used in predicting the path loss of various indoor propagation models using chronological backtrack algorithm, which is basic algorithm of CSP. After predicting the path loss at different set of parameters such as frequencies (f), floor attenuation factor (FAF), path loss coefficient (n), we find the optimum set of parameter frequency (f), floor attenuation factor (FAF), path loss coefficient(n) at which the path loss is minimum. The Branch and bound algorithm is used to optimize the constraint satisfaction problem.
\end{abstract}

Keywords: Path Loss, Indoor Propagation Model, CSP Algorithm, Attenuation Factor.

\section{INTRODUCTION}

Various research into adaptive algorithm has concerned to find the heuristics which is best suited for solving particular problems from a set of completely specified heuristics. In last few years, the constraint satisfaction programming (CSP) has attracted high attention among experts from many years because of its potential for solving problems. The constraint satisfaction programming approach has been widely used in many academics and research parlance to tackle wide range of search problem. It is defined by finite set of variables, a set of domain and constraints [1]. All CSPs are characterized by the inclusion of a finite set of variables; a set of domain values for each variable; and a set of constraints that are only satisfied by assigning particular domain values to the problem's variables [2]. The CSP deals with the set of values from its domain to the variable in order that no constraint is violated.

A CSP problem includes some variables, and valid values for those variables (we call it domain of the variables) and conflict tables. We must find a solution to assign values to all the variables and those values must satisfy the conflict tables [3]. There are currently two branches of constraint programming, namely constraint satisfaction and constraint solving.

Constraint satisfaction deals with the problem defined over finite domain, on the other hand constraint solving algorithm are based on mathematical techniques. The constraint satisfaction programming (CSP) offers its basic algorithm like backtracking and branch and bound algorithm to solve and optimize the problem. Constraints satisfaction algorithm can be viewed as an iterative procedure that repeatedly assigns domain value to the variables [4].

In this paper problem of finding the path loss of various Empirical indoor wireless propagation models in different environment has been stated as a CSP (constraint satisfaction problem) and has been solved by chronological backtracking algorithm. The branch and bound algorithm then used to optimize the constraint satisfaction problem.

Importance of propagation model is discussed in section II. In section III, methodology and basic algorithm of CSP is explained. Result of indoor model is discussed in section IV.

\section{PROPAGATION MODEL}

Nowadays cellular phones are used widely for the communication. The number of people using cell phone increases rapidly. Therefore, for an indoor environment an efficient planning and development is surely essential. For the design of indoor wireless services the knowledge of the signal propagation in different environment is demanded. The need for high capacity networks, estimating coverage accurately has become extremely important. Therefore, for more accurate design, signal strength measurement and the path loss measurement must be taken into consideration. Propagation models are used extensively in network planning, particularly for conducting feasibility studies and during initial deployment. Propagation models in wireless communication have focused on predicting the average received signal strength at a given distance from the transmitter as well as the variability of the signal strength in close proximity to a particular location. Propagation models that predict the mean signal strength for an arbitrary transmitter - receiver separation distance are useful in estimating the radio coverage area of transmitter. Propagation model that characterize the rapid fluctuation of the received signal strength over very short travel distances or short time duration are called small scale or fading models. As mobile moves over very small distances, the instantaneous received signal strength may fluctuate rapidly giving rise to small scale fading [5].

These models can be broadly categorized into three types: empirical, deterministic and stochastic. Empirical models are based on 
observation and measurement alone. These are mainly used to predict path loss [6]. Empirical models use measurement data to model a path loss equation. To conceive these models, a relationship is found between the received signal strength and other parameters such as distance, path loss exponent, floor loss attenuation factor. The main complexity to model indoor propagation channel is its sensitiveness to indoor environment and less probability of line-of-sight.

In this paper, the concept of constraint satisfaction programming has been implemented on indoor wireless propagation models in order to predicting and optimizing the propagation loss.

Two types of partitions in a building are very important hard and soft partitions. Hard partitions are immobile structures formed as a part of building. Soft partitions can be moved and do not span from the floor to the ceiling. One slope model is the simplest model for determining the path loss in indoor propagation. But it uses distance only to calculate the losses. It does not count floor attenuation, wall attenuation in calculation. It is found that penetration loss due to floor decreases as number of floors increase [5].

Two indoor models, distance dependent path loss model and floor attenuation factor path loss model have been developed. These models have been developed based on the number of floors between transmitter and receiver. The path loss depends upon different obstacles between transmitter and receivers.

\section{METHODOLOGY OF CSP}

Constraint satisfaction problems (CSPs) representing problems to form a class of models that have a common properties, a set of variables and a set of constraints. A solution to a CSP is a set of variable value assignments, which satisfies all members of the set of constraints in the CSP. In some situations, it is not possible to find a solution satisfy all the constraints belonging to a CSP. Such problems are termed as over constrained problems [7]. The algorithms or techniques that use in constraint satisfaction depend on the kind of constraint being considered. The two algorithms of CSP, chronological backtracking and branch and bound which are used to solve and optimize the wireless empirical propagation models are explained as below:-

Backtracking algorithm:

Backtracking is the basic algorithm to solve CSP. In every step, find a valid value to assign to current variable. If a valid value is found, assign it to current variable and go to next step. If there's no any valid value, back-track to the last variable to assign another value that can lead to the success of finding valid value for current variable [3]. The term backtracking search is used for a depth-first search that chooses values for one variable at a time and backtracks when a variable has no legal values left to assign. In context with the wireless empirical propagation model, we have different types of variables, such as frequency (f), distance (d), path loss exponent (n) etc. After implementation of the backtrack algorithm, we find the path loss of empirical propagation model at different set Frequency(f), distance (d), path loss exponent (n) and floor attenuation factor (FAF). Now after finding the path loss for different set of parameters, we have to find that particular set of parameter at which propagation loss is minimum. For this optimization CSP provides an optimization algorithm called branch and bound algorithm which may be explained as below.
Branch and Bound Algorithm:

A constraint satisfaction optimization problem (CSOP) is defined as CSP, together with an optimization function $\mathrm{f}$. So constraint satisfaction optimization problem is written as: $(\mathrm{X}, \mathrm{D}, \mathrm{C}, \mathrm{f})$ where $(\mathrm{X}$, D, C) represent CSP with a set of variables (X), domain (D) and constraints $(\mathrm{C})$ and $\mathrm{f}$ is the optimization function. A bound is nothing but a global variable which is defined according to the minimization or maximization problem, it depends upon the case that either problem needs minimum or maximum value of the function [4]. The branch and bound algorithm in empirical wireless propagation models is used to find that particular set of frequency(f), the distance (d), path loss exponent (n) and floor attenuation factor (FAF) at which propagation loss is minimum. After all the variables are labeled the calculated value of path loss is taken as the f value in branch and bound algorithm. This $\mathrm{f}$ value in branch and bound algorithm is compared with the estimated value of the global variable (bound), and if this computed $f$ value is less than the value of the existing bound, it will become the new bound. This procedure will carry on until and unless a minimum value is found and reverse of this procedure is used if we have to find the maximum value [4].

A constraint satisfaction problem is defined as tuple $\{\mathrm{X}, \mathrm{D}, \mathrm{C}\}$

where,

- $\quad \mathrm{X}$ is a finite set of variables,

- $\quad \mathrm{D}$ is a finite set of domains, one domain is assigned for each variable, and

- $\mathrm{C}$ is the finite set of constraints that restrict certain value assignments [8].

Domains of variables are: frequency, distance, path loss exponent and floor attenuation factor. Constraint is the path loss.

\section{RESULT \& DISCUSSION}

Among numerous propagation models, the following are the most significant ones. The indoor propagation models are:

i. ITU indoor propagation model

ii. Distance dependent path loss model

iii. Floor attenuation factor path loss model

i. ITU indoor propagation model:

Let us analyse the indoor propagation model by taking an example of ITU indoor propagation model. It is also known as ITU model for indoor attenuation. It is a radio propagation model that estimates the path loss inside a room or a closed area inside a building. This model is applicable for the frequency range of $900 \mathrm{MHz}$ to 5.2 $\mathrm{GHz}$. The ITU indoor path loss model is defined as,

$L=20 \log f+N \log d+P f(n)-28$

Where,

$\mathrm{N}$ is the distance power loss coefficient.

$\mathrm{n}$ is the number of floors between the transmitter and receiver. $\mathrm{Pf}(\mathrm{n})$ is the floor loss penetration factor 
For series 1:

$\begin{array}{llll}\text { f(MHz) } & \mathrm{d}(\mathrm{m}) & \mathrm{N} & \mathrm{Pf} \\ 915 & 1-5 & 1 & 30 \\ \begin{array}{l}\text { For series 2: } \\ \text { f(MHz) }\end{array} & \mathrm{d}(\mathrm{m}) & \mathrm{N} & \mathrm{Pf} \\ \begin{array}{l}\text { 915 } \\ \text { For series 3: }\end{array} & 1-5 & 1 & 33 \\ \text { f(MHz) } & \mathrm{d}(\mathrm{m}) & \mathrm{N} & \mathrm{Pf} \\ 1900 & 1-5 & 1 & 30\end{array}$

For series 4:

$\mathrm{f}(\mathrm{MHz}) \quad \mathrm{d}(\mathrm{m}) \quad \mathrm{N} \quad \mathrm{Pf}$

$\begin{array}{llll}2400 & 1-5 & 1 & 30\end{array}$

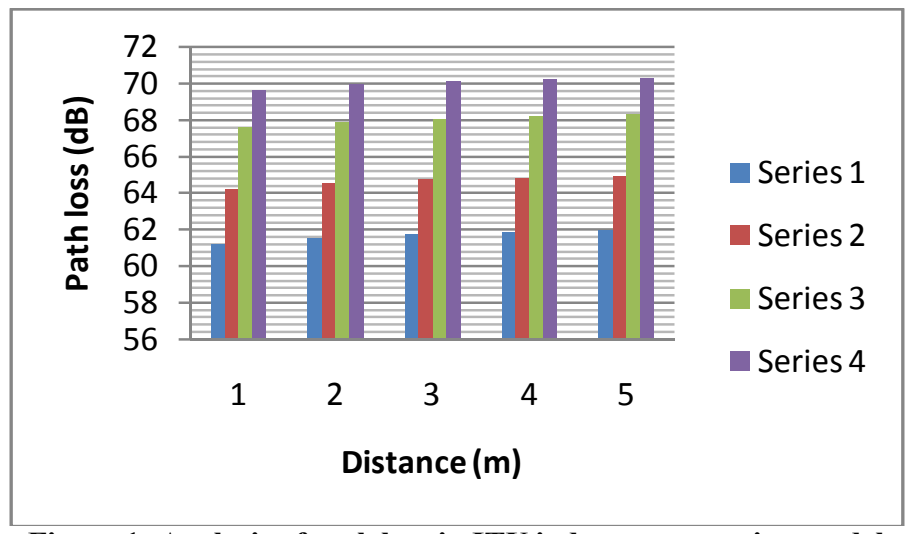

Figure 1- Analysis of path loss in ITU indoor propagation model

From the graphical analysis of figure 1 it can be concluded that the path loss is increased as the distance is increase, as well as floor loss penetration factor increase. It is clear that there is a high increment in the path loss when frequency is changes from $915 \mathrm{MHz}$ to 2400 $\mathrm{MHz}$.

ii. Distance dependent path loss model:

A model used in [9] shows that mean path loss increases exponentially with distance, i.e.,

$\overline{\mathrm{PL}}(\mathrm{d}) \propto\left(\frac{\mathrm{d}}{\mathrm{d}_{0}}\right) \mathrm{n}$

Where $\overline{\mathrm{PL}}$ is the mean path loss and $\mathrm{n}$ is the mean path loss exponent which indicates the path loss increases exponentially with distance. Absolute mean path loss in decibels is defined as the addition of the path loss at a reference distance $\mathrm{d} 0$ and some additional path loss in decibels.

$$
\overline{\mathrm{PL}}(\mathrm{d})[\mathrm{dB}]=\mathrm{PL}(\mathrm{d} 0)[\mathrm{dB}]+10 \mathrm{XnX} \log 10\left(\frac{\mathrm{d}}{\mathrm{d}_{0}}\right)
$$

For this, reference distance is chosen as $1 \mathrm{~m}$ and assume that $\mathrm{PL}(\mathrm{d} 0)$ is due to free space propagation from the transmitter to a $1 \mathrm{~m}$ reference distance. This leads to $31.67 \mathrm{~dB}$ path loss at $915 \mathrm{MHz}$ over a reference distance free space path. The value of $n$ depends on the specific propagation environment. For example, in free space, $n$ is equal to 2 , and when obstructions are present, $\mathrm{n}$ will have larger value. Table [5] lists path loss exponent obtained in different mobile radio environment.

Table 1. Path loss exponent in different mobile radio environment

\begin{tabular}{ll}
\hline Environment & $\begin{array}{c}\text { Path Loss } \\
\text { Exponent, } \boldsymbol{n}\end{array}$ \\
\hline Free space & 2 \\
Urban are cellular radio & 2.7 to 3.5 \\
Shadowed urban cellular radio & 3 to 5 \\
In building line-of-sight & 1.6 to 1.8 \\
Obstructed in building & 4 to 6 \\
Obstructed in factories & 2 to 3 \\
\hline
\end{tabular}

For series 1:

$\begin{array}{lll}\text { f(MHz) } & \mathrm{d}(\mathrm{m}) & \mathrm{n} \\ 915 & 1-5 & 2 \\ \begin{array}{l}\text { For series 2: } \\ \text { f(MHz) }\end{array} & \mathrm{d}(\mathrm{m}) & \mathrm{n} \\ 915 & 1-5 & 4 \\ \text { For series 3: } & & \\ \text { f(MHz) } & \mathrm{d}(\mathrm{m}) & \mathrm{n} \\ \text { 1900 } & 1-5 & 4 \\ \text { For series 4: } & & \\ \text { f(MHz) } & \mathrm{d}(\mathrm{m}) & \mathrm{n} \\ 2400 & 1-5 & 2\end{array}$

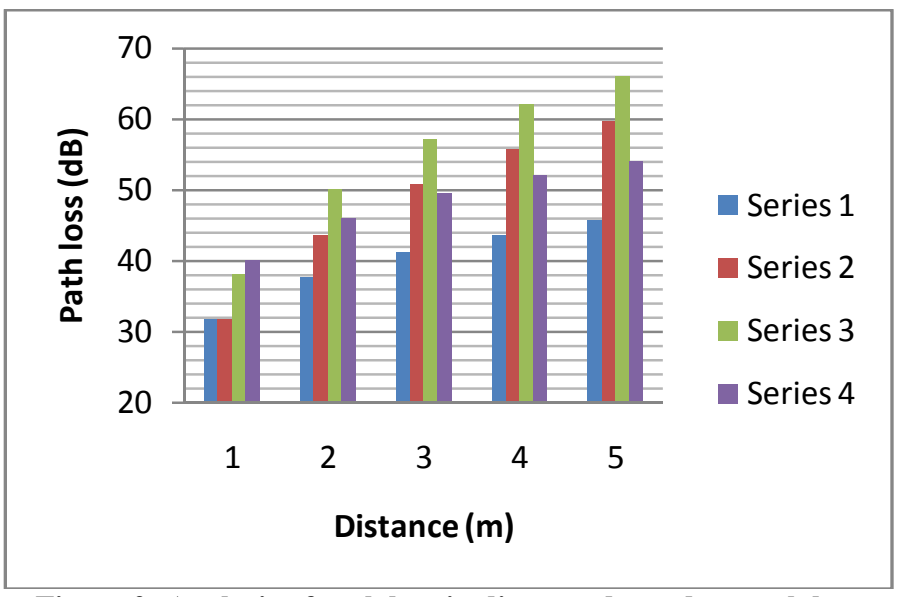

Figure 2- Analysis of path loss in distance dependent path loss model 
From the figure 2, it is clear that the mean path loss in distance dependent path loss model increases exponentially with distance. It can also be seen that it is depends on the path loss exponent. The value of $\mathrm{n}$ depends on the specific propagation environment.

\section{iii. Floor attenuation factor path loss model:}

The path loss in multi-floored environments is predicted by a mean path loss exponent that is a function of the number of floors between transmitter and receiver. The value of path loss exponent $n$ (multifloor) is taken from [5].

$$
\overline{\mathrm{PL}}(\mathrm{d})[\mathrm{dB}]=\mathrm{PL}(\mathrm{d} 0)[\mathrm{dB}]+10 \mathrm{X} \text { (multifloor) } X \log 10\left(\frac{\mathrm{d}}{\mathrm{d}_{0}}\right)
$$

The path loss in same floor environment is predicted by a constant floor attenuation factor (in dB). FAF is a function of the number of floors and building type. This FAF is basically added to the mean path loss predicted by a path loss model which uses the same floor path loss exponent for a particular building type. The value of path loss exponent $\mathrm{n}$ (same floor) is taken from [5].

$\overline{\mathrm{PL}}(\mathrm{d})[\mathrm{dB}]=\mathrm{PL}(\mathrm{d} 0)[\mathrm{dB}]+10 \mathrm{X}$ (same floor) $\mathrm{X} \log 10\left(\frac{\mathrm{d}}{\mathrm{d}_{0}}\right)+\mathrm{FAF}[\mathrm{dB}]$ (5)

The attenuation between one floor of the building is greater than the incremental attenuation caused by each additional floor. The average attenuation factors for an identical number of floors between the transmitter and receiver for the two environments differ by 3-8 dB [9].

There are two examples of measuring the path loss with the use of two different models through two floors and three floors building respectively.

For two floors building, the mean path loss exponent for same floor measurement is $\mathrm{n}=3.27$ and the average floor attenuation factor is $\mathrm{FAF}=18.7$, the mean path loss exponent for two-floor measurement is $\mathrm{n}=5.04$. The frequency and reference distance is chosen as $915 \mathrm{MHz}$ and $1 \mathrm{~m}$ respectively. Then at a seperation of $d=30 \mathrm{~m}$, the predicted path loss is

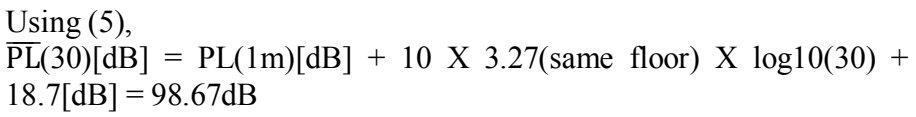

Or using (4),

$\overline{\mathrm{PL}}(30)[\mathrm{dB}]=\mathrm{PL}(1 \mathrm{~m})[\mathrm{dB}]+10 \mathrm{X} 5.04$ (multifloor) $\mathrm{X} \log 10(30)=$ $106.12 \mathrm{~dB}$

For three floors building, the mean path loss exponent for same floor measurement is $\mathrm{n}=3.27$ and the average floor attenuation factor is $\mathrm{FAF}=24.4$, the mean path loss exponent for three-floor measurement is $n=5.22$. The frequency and reference distance is chosen as $915 \mathrm{MHz}$ and $1 \mathrm{~m}$ respectively. Then at a seperation of $\mathrm{d}=$ $30 \mathrm{~m}$, the predicted path loss is

Using (5),
$\overline{\mathrm{PL}}(30)[\mathrm{dB}]=\mathrm{PL}(1 \mathrm{~m})[\mathrm{dB}]+10 \mathrm{X} 3.27$ (same floor) $\mathrm{X} \log 10(30)+$ $24.4[\mathrm{~dB}]=104.37 \mathrm{~dB}$

Or using (4),

$\overline{\mathrm{PL}}(30)[\mathrm{dB}]=\mathrm{PL}(1 \mathrm{~m})[\mathrm{dB}]+10 \mathrm{X} 5.22$ (multifloor) $\mathrm{X} \log 10(30)=$ $108.78 \mathrm{~dB}$

So it is clear from the above explanation that the average attenuation factors for an identical number of floors between the transmitter and receiver for the two buildings differ by 3-8 dB.

\section{COMPARISON BETWEEN INDOOR MODELS}

In ITU indoor model, as the frequency increases the path loss also increases. Initially losses are high at high frequency but as the partition increases the loss rate is low as compared to at low frequency. The more partition causes the less loss rate. Because of that there is not much variation in lsss for far end partition. The distance dependent path loss model is basically relying on the environment we are working in, because there is a different path loss exponent for a different indoor environment. This model is not suitable if we are taking a wall effect or floor effect. Floor attenuation factor path loss model is the most appropriate model which can be used in predicting the path loss in indoor environment. It uses the two different models mutifloor and same floor environment. Both have different mean path loss exponent. Floor attenuation factor model is most affected by partitions. The attenuation between one floor of the building is greater than the incremental attenuation caused by each additional floor. So it is useful for multi floor building, mainly for the building having more than three floors.

\section{CONCLUSION}

In the first part of this paper, I briefly introduced about constraint satisfaction, which provides a general basis for constraint satisfaction algorithm. In this paper the various wireless empirical propagation model has been solved at $915 \mathrm{MHz}$ in different environment to find the path loss using the constraint satisfaction algorithm. The models are based on a simple dn exponential path loss vs. distance relationship. I also discussed the main technique to solve CSP, constraint satisfaction optimization algorithm to optimize the different empirical propagation models to find the parameters frequency, distance, path loss exponent, floor attenuation factor at which the path loss is minimum. The models have been shown to be more accurate when considering the different buildings and different environment within the same building separately

\section{REFERENCES}

[1] Tope R. Karem, H. Anthony Chan, "A low cost design of next generation sonnet/sdh network with multiple constraint", IEEE, National Research Foundation, 2007.

[2] Stuart Bain, John Thornton, Abdul Sattar, "Evolving algorithm for constraint satisfaction”, IEEE, pp. 265-272, 2004. 
[3] Tianbing Lin, Scott Goodwin, "CSP: definition, creation, and algorithms".

[4] Nagendra Sah and Amit Kumar, "CSP algorithm in predicting and optimizing the path loss of wireless empirical propagation models", International Journal of Computer and Electrical Engineering, vol. 1, no. 4, pp. 464-472, October 2009.

[5] Theodore S. Rappaport, "Wireless communications: principles and practice", Second Eddition, PHI Learning Private Limited, 2008.

[6] V.S. Abhayawardhana, I.J. Wassell, D. Crosby, M.P. Sellars, M.G. Brown, "Comparison of Empirical propagation path Loss Models for Fixed Wireless Access Systems”, IEEE, 2005.

[7] Srinivas Padmanabhuni, "Extended analysis of intelligent backtracking algorithms for the maximal constraint satisfaction problem", In the proceedings of the 1999 IEEE Canadian Conference on Electrical and Computer Engineernig, pp. 17101715, May 1999.

[8] J.I. van Hemert, "Evolving binary constraint satisfaction problem instances that are difficult to solve", National Research Institute for Mathematics and Computer Science, IEEE, pp. 1267-1273, 2003.

[9] Scott Y. Seidel, Theodore S. Rappaport, "914 MHz path loss prediction models for indoor wireless communications in multifloored buildings", In the proceeding of IEEE Transactions on Antennas and Propagation, vol. 40, no. 2, pp. 207-217, Feb 1992 